\title{
Neurological soft signs in mainstream pupils
}

\author{
J M Fellick, A P J Thomson, J Sills, C A Hart
}

\begin{abstract}
Aims-(1) To examine the relation between neurological soft signs and measures of cognition, coordination, and behaviour in mainstream schoolchildren. (2) To determine whether high soft sign scores may predict children with significant problems in other areas.

Methods-A total of 169 children aged between 8 and 13 years from mainstream schools were assessed. They form part of a larger study into the outcome of meningococcal disease in childhood. Half had previous meningococcal disease and half were controls. Assessment involved measurement of six soft signs followed by assessment of motor skills (movement ABC), cognitive function (WISC-III), and behaviour (Conners' Rating Scales). Results-Children having an age corrected soft sign score above the 90th centile were considered to have an excess of soft signs. When compared to the other children they had significantly worse scores on the other three measures. Median movement ABC score was $15.3 v$ 7. Mean total IQ scores were lower by 10.3 points. Median behaviour scores were significantly higher on both parental and teacher questionnaires. A soft sign score above the 90th centile had a sensitivity of $38 \%$ for detecting cognitive impairment, $42 \%$ for detecting coordination problems, and $25 \%$ for detecting possible attention deficit hyperactivity disorder.

Conclusion-In this group of children higher scores on the soft sign battery were related to significantly worse performance on measures of cognition, coordination, and behaviour. However, although soft sign assessment may be of interest it cannot accurately predict which children are likely to have impairment in other areas. (Arch Dis Child 2001;85:371-374)
\end{abstract}

Keywords: neurological soft signs; ADHD; learning difficulties; developmental coordination disorder

Paediatricians in the UK are increasingly being asked to assess children in mainstream school who are not performing as well as their peers. Differential diagnoses include developmental coordination disorder, specific or general learning difficulties, and behaviour problems such as attention deficit hyperactivity disorder (ADHD). Frequently children may have problems in all three areas. Neurological examination is usually normal and subsequent assessment is time consuming and may involve multiple agencies such as education, occupational therapy, and child and adolescent psychiatry. Are there any tests that a paediatrician may use to predict which children have significant problems?

Neurological soft signs (NSS) may be defined as minor abnormalities in the neurological examination in the absence of other features of fixed or transient neurological disorder. ${ }^{1}$ They have been associated with behaviour, ${ }^{12}$ coordination, ${ }^{3}$ and learning difficulties. ${ }^{4}$ Other authors believe they represent a developmental lag rather than a fixed abnormality. ${ }^{5}$ Studies have found a high incidence of soft signs in children following premature ${ }^{6}$ or low birthweight ${ }^{7}$ birth, meningitis, ${ }^{8}$ and malnutrition. ${ }^{9} 10$

There are a number of soft sign batteries published that include tests of sensory function, coordination, motor speed, and abnormal or associated movements. ${ }^{11}{ }^{12}$ Some have been validated and tested longitudinally. ${ }^{12-14}$ Tests may be performed quickly in the clinic situation and do not require special equipment.

We aim firstly to examine the associations between NSS and measures of cognitive ability, motor performance, and behaviour in a group of mainstream school children; and secondly to determine whether NSSs may be used to predict significant problems in other areas.

\section{Methods}

SUBJECTS

A total of 169 children, aged between 8 and 13, attending mainstream school in Merseyside were assessed. They were part of a larger study into the neurodevelopmental outcomes of meningococcal disease (MCD) in childhood. Half had suffered previous MCD and half were controls.

\section{ASSESSMENTS}

These took place at the Royal Liverpool Children's Hospital (RLCH) or at school, depending on preference. All tests were performed by the research fellow following appropriate training. Written consent was obtained prior to testing. Approval was gained from the RLCH and local research and ethics committees. Testing took approximately 1.5 hours to complete and was performed in the same order throughout.

\section{Neurological examination}

A standardised neurological examination of cranial and peripheral systems was performed including assessment of power, tone, and reflexes. Any children with focal neurological signs were excluded from the soft sign analysis.

\section{Soft sign assessment}

Six neurological soft signs were assessed following the protocol devised by Shafer and 
Table 1 Raw data

\begin{tabular}{|c|c|c|c|c|c|c|}
\hline & Stereognosis & Graphaesthesia & Dysdiadochokinesis & $\begin{array}{l}\text { Mirror } \\
\text { movements }\end{array}$ & Motor speed & $\begin{array}{l}\text { Involuntary } \\
\text { movements }\end{array}$ \\
\hline Median & 0 & 0 & 6 & 4 & 72 & 0 \\
\hline Interquartile range (IQR) & $0-0$ & $0-1$ & $3-8$ & $2-6$ & $64-80$ & $0-1$ \\
\hline Range & $0-3$ & $0-3$ & $0-14$ & $0-12$ & $47-145$ & $0-4$ \\
\hline $\begin{array}{l}\text { Spearman correlation to } \\
\text { total score }\end{array}$ & 0.24 & 0.51 & 0.65 & 0.61 & 0.69 & 0.64 \\
\hline
\end{tabular}

colleagues. ${ }^{12}$ The signs assessed were stereognosis, graphaesthesia, dysdiadokokinesis, mirror movements, motor speed, and involuntary movements. For each subject scores were obtained for each individual soft sign and a total summary score was calculated. High scores indicate more soft signs.

\section{The Movement $A B C^{15}$}

This is a battery of tests designed to assess motor and coordination skills in children. The test involves eight tests of motor function (three of manual dexterity, two of ball skills, and three of static and dynamic balance). The battery is age standardised and results in an overall impairment score between 0 and 40, with high scores indicating poorer function. Scores above the 95 th centile are considered to indicate definite motor problems.

The Wechsler Intelligence Scale for Children, third edition UK (WISC-III UK $)^{16}$

The first eight subtests of the WISC-III ${ }^{\mathrm{UK}}$ were administered. Verbal (VIQ), performance (PIQ), and total IQ (TIQ) scores were calculated and expressed as standardised scores (mean 100, SD 15).

Test of visual-motor integration (VMI $)^{17}$

This test evaluates the ability to copy a sequence of geometric forms of increasing

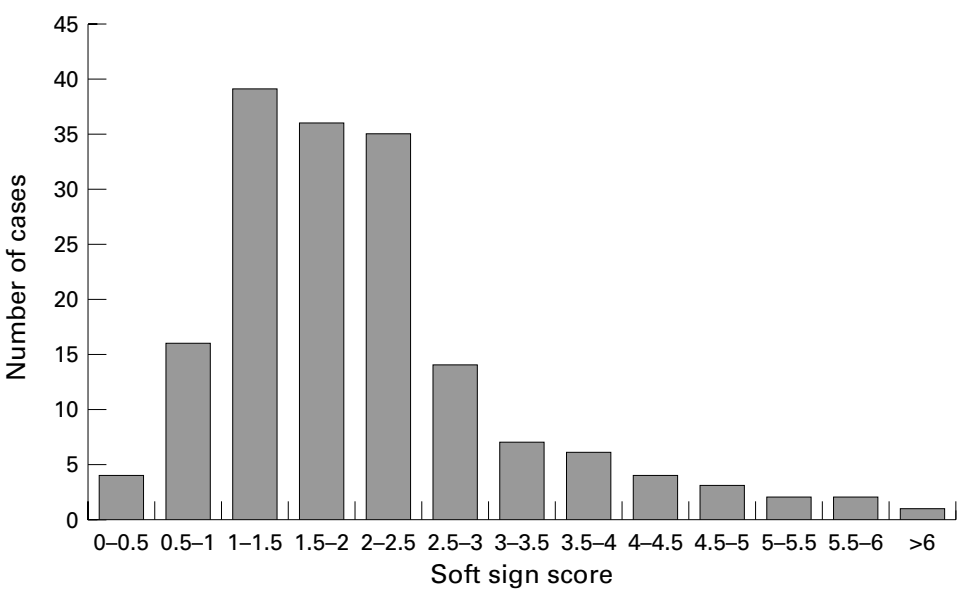

Figure 1 Frequency histogram of soft sign scores.

Table 2 Relation between coordination skills and soft signs

\begin{tabular}{llll}
\hline Movement $A B C$ & $\begin{array}{l}\text { Normal } \\
(n=151)\end{array}$ & $\begin{array}{l}\text { High soft signs } \\
(n=18)\end{array}$ & pvalue $^{*}$ \\
\hline Manual dexterity, median (IQR) & $4.5(2.0-7.0)$ & $10.0(7.3-11.4)$ & 0.000 \\
Ball skills, median (IQR) & $1.0(1.0-3.0)$ & $3.0(1.0-5.3)$ & 0.004 \\
Balance, median (IQR) & $1.5(0.0-3.0)$ & $3.8(1.4-6.8)$ & 0.003 \\
Total, median (IQR) & $7.0(4.0-11.0)$ & $15.3(11.1-23.1)$ & 0.000 \\
\hline
\end{tabular}

^Mann-Whitney U test. complexity. Raw scores are age corrected and expressed as standardised scores (mean 100, SD 15).

\section{Behaviour}

The long form of Conners' Rating Scales, Revised (CRS-R) ${ }^{18}$ was administered to the child's parent/guardian and to their teacher. These scales are designed to assess ADHD and related behavioural problems. The questions relate directly to the Diagnostic and Statistical Manual of Mental Disorders, Fourth Edition (DSM IV). Age standardised t scores (mean 50, SD 10) are produced for each behaviour type, with scores over 70 indicating a possible problem.

\section{Demographics}

In addition to the behaviour scales, parents completed demographic questionnaires to determine social class, family size, and type of housing (owned/rented) for each case.

\section{STATISTICAL ANALYSIS}

Age adjusted total soft sign scores were calculated for each case. Subjects with scores above the 90th centile were considered to have an excess of soft signs. Bivariate analysis compared these cases to the remainder using either the $t$ test for independent samples or the Mann-Whitney $U$ test, depending upon the level of data. Analysis was performed on SPSS.

\section{Results}

SOFT SIGN DATA

Table 1 summarises the raw soft sign data available. The sensory soft signs (stereognosis and graphaesthesia) were rarely present and have lower correlations to total soft sign score. Involuntary movements were also rarely seen, but when present correlated highly with total score. Total soft signs scores were inversely related to age in a linear fashion (Pearson coefficient $-0.4, p=0.01)$. Scores were therefore age corrected using linear regression.

Figure 1 shows a histogram of corrected soft sign scores. Soft sign scores up to 3.5 were normally distributed. Cases with scores greater than 3.5 were considered to have an excess of soft signs ( $\mathrm{n}=18,10 \%$ of total). There were no significant demographic differences in terms of social class, family size, and type of housing between those with high and normal soft sign scores.

RELATION TO MOVEMENT ABC

Table 2 compares median movement ABC scores for patients with and without excess soft signs. Patients with high soft sign scores scored 
Table 3 Relation between measures of cognitive function and soft signs

\begin{tabular}{lllll}
\hline & $\begin{array}{l}\text { Normal } \\
(n=151)\end{array}$ & $\begin{array}{l}\text { High soft signs } \\
(n=18)\end{array}$ & $\begin{array}{l}\text { Difference } \\
(95 \% \text { CI })\end{array}$ & p value $^{*}$ \\
\hline Mean VMI (SD) & $98.3(11.6)$ & $91.7(15.7)$ & $6.6(0.6-12.5)$ & 0.03 \\
Mean VIQ (SD) & $98.2(15.8)$ & $84.8(13.6)$ & $13.4(5.7-21)$ & 0.01 \\
Mean PIQ (SD) & $98.4(14.7)$ & $83.9(11.7)$ & $14.5(7.4-22)$ & 0.00 \\
Mean TIQ (SD) & $98.1(15.4)$ & $83.1(12.5)$ & $15(8.3-20.7)$ & 0.00 \\
\hline
\end{tabular}

${ }^{\star} t$ test.

VMI, developmental test of visual-motor integration; VIQ, verbal intelligence quotient; PIQ, per-

formance intelligence quotient; TIQ, total intelligence quotient.

Table 4 Parental behaviour scores

\begin{tabular}{llll}
\hline & $\begin{array}{l}\text { High soft signs } \\
(n=18)\end{array}$ & $\begin{array}{l}\text { Normal } \\
(n=148)\end{array}$ & pvalue $^{*}$ \\
\hline Oppositional, median t score (IQR) & $60(45-79)$ & $50(45-60)$ & 0.06 \\
Anxious, median t score (IQR) & $57(47-64)$ & $48(44-55)$ & 0.1 \\
Social problems, median t score (IQR) & $50(49-62)$ & $45(45-53)$ & 0.001 \\
Global problems, median t score (IQR) & $65(54-77)$ & $51(44-61)$ & 0.001 \\
ADHD, median t score (IQR) & $62(54-79)$ & $50(45-59)$ & 0.001 \\
\hline
\end{tabular}

*Mann-Whitney U test.

ADHD, attention deficit hyperactivity disorder.

Table 5 Teacher behaviour scores

\begin{tabular}{llll}
\hline & $\begin{array}{l}\text { High soft signs } \\
(n=16)\end{array}$ & $\begin{array}{l}\text { Normal } \\
(n=136)\end{array}$ & $p^{\text {value* }}$ \\
\hline Oppositional, median t score (IQR) & $47(45-66)$ & $46(45-55)$ & 0.78 \\
Anxiety, median t score (IQR) & $59(49-66)$ & $51(46-62)$ & 0.21 \\
Social problems, median t score (IQR) & $50(46-76)$ & $46(45-51)$ & 0.05 \\
Global problems, median t score (IQR) & $57(49-76)$ & $52(44-60)$ & 0.03 \\
ADHD, median t score (IQR) & $59(49-69)$ & $50(43-59)$ & 0.01
\end{tabular}

*Mann-Whitney U test.

ADHD, attention deficit hyperactivity disorder.

significantly higher on all three subsets and total score on the movement ABC. The Pearson correlation coefficient between movement ABC score and soft sign score was 0.36 $(\mathrm{p}=0.01)$. Subjects with a movement ABC score above 17 ( $>98 \%$ on standardisation data) were classified as having significant coordination difficulties $(n=19)$. Soft sign score above 3.5 detected these cases with a sensitivity of $42 \%$ and a positive predictive value of $44 \%$.

RELATION TO COGNITIVE MEASURES

Table 3 shows that patients with high soft sign scores performed less well on all measures of cognitive function. Total IQ scores were lower

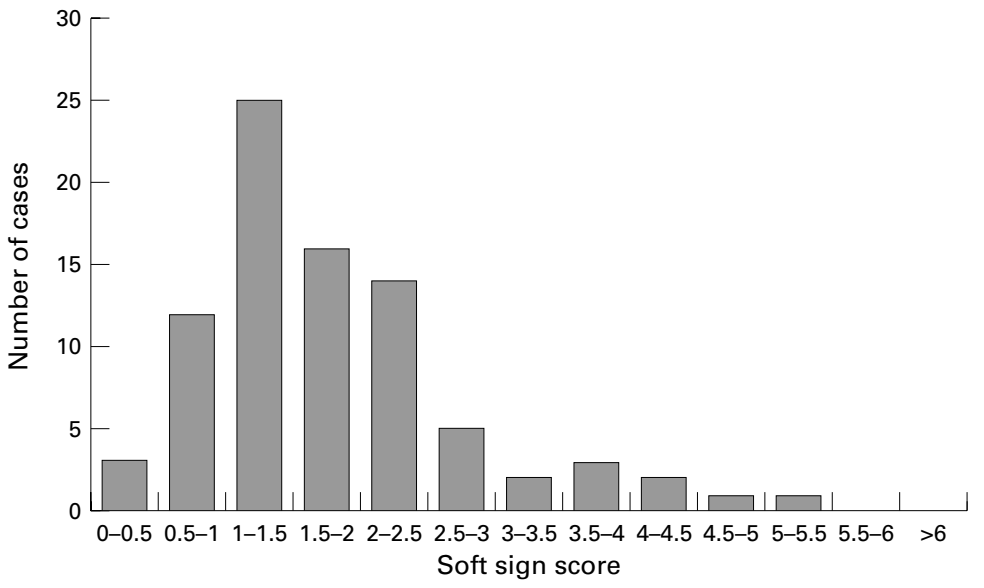

Figure 2 Frequency histogram of soft sign scores for controls only. by 15 points ( $95 \%$ CI 8.3 to 20.7 ), with similar differences for verbal and performance scores and VMI. The Spearman correlation coefficient between total IQ and soft sign score was $0.3(\mathrm{p}=0.01)$.

Subjects with a total IQ score below 75 ( $<98 \%$ on standardisation data) were classified as having significant learning difficulties $(n=16)$. A soft sign score above 3.5 detected these cases with a sensitivity of $38 \%$ and a positive predictive value of $33 \%$.

RELATION TO BEHAVIOUR

Tables 4 (parents) and 5 (teachers) show median scores for five behaviours. Subjects with excess soft signs scored significantly higher on parental scales for oppositional, social, global, and ADHD problems. Spearman correlation coefficient was $0.23(\mathrm{p}=0.01)$, between global t score and soft sign score.

On teacher scales significant differences were found for social, global, and ADHD problems; cases with excess soft sign scores had higher behaviour scores. Spearman correlation coefficient was $0.17(\mathrm{p}=0.05)$, between global $\mathrm{t}$ score and soft sign score.

Subjects with a teacher score above 75 for global problems $(>98 \%$ on standardisation data) were classified as having significant behaviour problems $(n=16)$. A soft sign score above 3.5 detected these cases with a sensitivity of $25 \%$ and a positive predictive value of $25 \%$.

\section{Discussion}

We have shown that in a cohort of mainstream school children aged between 8 and 13, those with high ( $>90$ th centile) soft sign scores performed significantly worse on tests of coordination, cognition, and behaviour. However, soft sign assessment alone was not sufficiently sensitive in predicting significant problems in other areas to make this a useful clinical tool.

This study involved a large number of children. The fact that half had suffered previous MCD should not affect the aims to establish associations between NSSs and other measures. Analysis of the controls alone showed the same relation between high soft sign scores and lower performance on the other measures. Figure 2 shows the frequency distribution for corrected soft sign scores for controls alone and is similar to fig 1 . Of the 18 cases defined as having excess soft signs, 11 had previous MCD and seven were controls $\left(\mathrm{p}=0.33, \chi^{2}\right)$.

The NSS battery used has been subject to reliability and longitudinal studies and has been widely used in research. ${ }^{12} 1419$ The Movement ABC, Wechsler Scales, VMI, and Conners' Scales are all widely used in both research and clinical practice. Testing was performed by a single research fellow, eliminating any intertester bias. Tests were performed in the same order throughout. Although the soft sign tests were administered first, the total scores were not calculated and age adjusted until after the completion of all tests. 
This study again shows the association between NSSs and age with scores being lower in older children. This supports the theory that NSSs represent a developmental phenomenon. However, following correction for age we have shown an association between high soft sign scores and poorer performance on tests of coordination, cognition, and behaviour. This supports the earlier work of many authors who hypothesised that neurological soft signs represent a fixed neurological deficit. It is likely that both developmental and neurological factors together determine the level of soft signs in an individual.

Are NSSs a useful clinical tool for paediatricians? The battery used needed minimal training and took about five minutes to perform. The two sensory tests contributed little to the overall score and could be excluded, making testing even quicker. Apart from a stopwatch, no additional equipment is needed. From a practical aspect therefore, this test is quick and easy to perform. However, how would we interpret scores in the clinical setting? From our results a child with a high soft sign score is more likely to have significant problems with coordination, cognition, and behaviour than one with a lower score. However, the test is not sensitive or specific enough in detecting children with significant problems in other areas. Although soft sign assessment may be of interest to paediatricians, it gives little information to guide further management.

1 Shaffer D, OConnor PA, Shafer SQ. Neurological soft signs: their origins and significance for behaviour. In: Rutter M, ed. Developmental neuropsychiatry. New York: Guildford ed. Developmental neurc

2 Pine DS, Wasserman GA, Fried JE, et al. Neurological soft signs: one-year stability and relationship to psychiatric symptoms in boys. F Am Acad Child Adolesc Psychiatry 1997;36:1579-86.

3 Henderson SE, Hall D. Concomitants of clumsiness in young schoolchildren. Dev Med Child Neurol 1982;24:44860.

4 Adams RM, Kocsis JJ, Estes RE. Soft neurological signs in learning - disabled children and controls. Am $\mathcal{f}$ Dis Child 1974;128:614-18.

5 Blondis TA, Snow JH, Accardo PJ. Integration of soft signs in academically normal and academically at-risk children. Pediatrics $1990 ; 85$ (3 Pt 2):421-5.

6 Jongmans M, Mercuri E, de Vries L, et al. Minor neurological signs and perceptual-motor difficulties in prematurely born children. Arch Dis Child Fetal Neonatal Ed 1997;76: F9-14.

7 Hertzig ME. Neurological "soft" signs in low-birthweight children. Dev Med Child Neurol 1981;23:778-91.

8 Grimwood K, Anderson VA, Bond L, et al. Adverse outcomes of bacterial meningitis in school-age survivors. outcomes of bacterial menin
Pediatrics 1995;95:646-56.

9 Galler JR, Ramsey F, Solimano G, et al. The influence of early malnutrition on subsequent behavioral development. IV. Soft neurologic signs. Pediatr Res 1984;18:826-32.

10 Galler JR, Ramsey F, Solimano G. A follow-up study of the effects of early malnutrition on subsequent development. II. Fine motor skills in adolescence. Pediatr Res 1985;19: 524-7.

11 Camp JA, Bialer I, Press M, Winsberg BG. The physical and neurological examination for soft signs (PANESS) and neurological examic norms and comparisons between nANESS) pediatric norms and comparisons between normal and 39-41.

12 Stokman CJ, Shafer SQ, Shaffer D, et al. Assessment of neurological "soft signs" in adolescents: reliability studies. Dev Med Child Neurol 1986;28:428-39.

13 Holden EW, Tarnowski KJ, Prinz RJ. Reliability of neurological soft signs in children: reevaluation of the neurological soft signs in children: reevaluation
PANESS. $\mathcal{F}$ Abnorm Child Psychol 1982;10:163-72.

14 Shafer SQ, Stokman CJ, Shaffer D, et al. Ten-year consistency in neurological test performance of children without focal neurological deficit. Dev Med Child Neurol 1986;28:417-27.

15 Henderson SE, Sugden DA. Movement assessment battery for children. London: The Psychological Corporation Ltd, 1992.
16 Wechsler D. Wechsler Intelligence Scale for Children, third edition, UK. Sidcup: The Psychological Corporation Ltd, edition,

17 Beery KE. The Beery-Buktenica Developmental Test of VisualMotor Integration. Cleveland: Modern Curriculum Press, 1997

18 Conners K. Conners' Rating Scales, Revised. North Tonawanda, NY: Multi-Health Systems Inc., 1997.

19 Pine DS, Scott MR, Busner C, et al. Psychometrics of neurological soft signs. F Am Acad Child Adolesc Psychiatry 1996;35:509-15.

Commentary-soft signs: soft neurologist The editor has asked me to give my opinion on soft neurological signs. I understand that I need not apply the techniques of meta-analysis or any sort of evidence based medicine. Wonderful. However, he asked me to write so as to be understood by foreign readers. It is good that I write instead of speak. Before I gave a paper at a Rett syndrome meeting in Vienna I told the conference an anecdote: a paediatric neurologist from Ljubljana had told me that she only understood $50 \%$ of what I said, but I replied that this was better than in Glasgow; so, could my paper please be translated simultaneously into both German and into English.

Before his knighthood, Peter Tizard was reported to have said something to the effect that training is for dogs, acrobats, and surgeons. Insofar as I may use that term, I had the great good fortune to do my training in paediatric neurology at the Hospital for Sick Children, Toronto. Not only that, but I had the privilege to spend the greatest part of my fellowship under the tutelage of John Steele, eponymously immortal in the SteeleRichardson-Olszewski syndrome. ${ }^{1} \mathrm{He}$ was and is predominantly an adult neurologistbut one who has made a substantial contribution to paediatric neurology - and one of his greatest gifts was to instil in me the importance of hard rather than soft neurological signs. As the head of that department, John Stobo Prichard emphasised the adage ${ }^{2}$ what's the lesion-history, where's the lesionexamination. Although the neurological examination of the child is often predominantly observational and unsystematic, ${ }^{3}$ a major objective of the examination is localisation, and localisation comes from hard signs, not soft.

It is a great pleasure to see a paper which concludes that soft signs give paediatricians little information. Much more useful would be to enhance one's real hard neurological skills, from how to elicit tendon reflexes in a neonate ${ }^{4}$ to how to tap the head of a child with headache for MacEwen's sign. Soft neurological signs should be consigned to the dustbin - or is it recycle bin-of paediatric history.

PROF. JOHN B P STEPHENSON 27 Charles Crescent, Lenzie, Glasgow G66 5HH, UK

1 Steele JC. Progressive supranuclear palsy. Historical notes. J Neural Transm Suppl 1994;42:3-14.

2 Stephenson JBP. Fits and faints. London: MacKeith Press, 1990:10.

3 Aicardi J. Disease of the nervous system in childhood. London: MacKeith Press, 1992:xii.

4 Stephenson JBP. Neonatal presentation of Prader-Willi syndrome. Am F Dis Child 1992;146:151-2. 